\title{
Delayed Lateral Row Anchor Failure in Suture Bridge Rotator Cuff Repair: A Report of 3 Cases
}

\author{
Jae-Jung Jeong, Jong-Hun Ji , Seok-Jae Park \\ Department of Orthopedic Surgery, Daejoen St. Mary's Hospital, College of Medicine, The Catholic University of Korea, Daejoen, Korea
}

\begin{abstract}
Compared to single row repair, use of lateral row anchors in suture bridge rotator cuff repair enhances repair strength and increases footprint contact area. If a lateral knotless anchor (push-in design) is inserted into osteoporotic bone, pull-out of the lateral row anchor can developed. However, failures of lateral row anchors have been reported at several months after surgery. In our cases, even though complete cuff healing occurred, delayed pull-out of the lateral row anchor in the suture bridge repair occurred. In comparison to a conventional medial anchor, further biomechanical evaluation of the pull-out force, design, and insertion angle of the lateral anchor is needed in future studies. We report three cases with delayed pull-out of lateral row anchor in suture bridge rotator cuff repair with a literature review.
\end{abstract}

(Clin Shoulder Elbow 2018;21(4):246-251)

Key Words: Lateral row anchors; Suture bridge rotator cuff repair; Pull-out; Repair strength; Footprint

The suture bridge repair is a reliable method of recreating the cuff footprint to be biomechanically stronger; this technique offers higher structural integrity and has been commonly used in rotator cuff repair instead of the single row repair. However, several demerits of suture bridge repair include increased surgical time and cost, and the possibility for stress overload of the repair due to the excessive lateralization of the residual tendon. ${ }^{1)}$

Failure of the medial or lateral row anchor is rarely reported in suture bridge repair. A previous study reported that the load borne by the lateral row for suture bridge repair was similar to the medial row. ${ }^{2)}$ In our cases, patients unintentionally experienced early mobilization, and pull-out of the lateral row anchor then developed several months after rotator cuff repair. Anchor pull-out is generally more likely to develop in osteoporotic bone and the suture anchor should be placed in the proximal anterior and middle parts of the greater tuberosity. ${ }^{3)}$ However, our patients did not show any evidence of osteoporosis. In this type of failure, the type of suture anchor, the pull-out strength of the suture anchor, the anchor insertion angle in the medial and lat- eral row, and the rehabilitation after rotator cuff repair should be considered.

The purpose of this study was to report three cases of lateral row anchor failure in conventional suture bridge repair, to evaluate the clinical results, and to consider the mechanism of lateral row anchor failure with a literature review. We hypothesized that the one of several causes of delayed shoulder pain after rotator cuff suture bridge repair was late lateral row anchor pullout, and magnetic resonance imaging (MRI) or ultrasound evaluation should be considered for differential diagnosis.

\section{Case Report}

Rotator cuff repair was performed among 752 patients from March 2008 to March 2011. Among these patients, three patients who underwent arthroscopic suture bridge repair in the rotator cuff tear showed lateral row anchor failure. These three patients were retrospectively evaluated for clinical and radiological outcomes. In these patients, we performed arthroscopic su-

Received September 5, 2018. Revised October 8, 2018. Accepted October 10, 2018.

Correspondence to: Jong-Hun Ji

Department of Orthopedic Surgery, Daejeon St. Mary's Hospital, College of Medicine, The Catholic University of Korea, 64 Daeheung-ro, Junggu, Daejeon 34943, Korea

Tel: +82-42-220-9530, Fax: +82-42-221-0429, E-mail: jijh87@gmail.com, ORCID: https://orcid.org/0000-0003-1087-3254

IRB approval: The Catholic University of Korea, Daejeon St. Mary's Hospital (IRB No: DC18ZESI0088).

Financial support: None. Conflict of interests: None. 
ture bridge repair. There were two male patients and one female patient with an average age of 62 years (range: 54-74 years). The mean duration between initial operation and revision operation was 4.7 months (range: 3-6 months). The tear pattern of the torn cuff was a high-grade, bursal side, partial thickness rotator cuff tear in one patient, concurrent bursal and articular side partial thickness rotator cuff tear in one patient, and largesized, full thickness rotator cuff tear in one patient. In partial thickness tears, if the mediolateral or anteroposterior tear size of the partial tear is more than $1 \mathrm{~cm}$, we prefer suture bridge rotator cuff repair. In the failure cases, only one patient had a trauma history (falling from a bicycle). None of the patients were taking osteoporosis medications and all patients showed normal bone mineral density findings. An experienced doctor measured the range of motion (ROM) of both shoulders using a goniometer. Institutional review board approval was obtained before retrieval of any data.

\section{Case 1}

A 75-year-old male patient had chronic right shoulder pain. MRI showed high-grade partial thickness rotator cuff tear in the shoulder and arthroscopic findings revealed $1.5 \times 1.0 \mathrm{~cm}$ highgrade bursal side partial thickness rotator cuff tear. Type II supe- rior labrum anterior and posterior (SLAP) lesion was found and debridement was performed. Arthroscopic suture bridge repair was performed. In the medial row, one metal anchor with two threads (5.0 mm Twin-Fix anchor; Smith-Nephew, Andover, MA, USA) was inserted near the articular margin of the humeral head. Four strands from one medial anchor we passed through the torn cuff and tied in a horizontal mattress pattern. One of two limbs from one medial anchor was pulled laterally over the tendon. After the lateral bone was prepped for anchor placement, the two limbs were passed through the eyelet of a knotless anchor (Versalock; Depuy Mitek, Raynham, MA, USA) and compressed the repaired cuff. This repair was performed in an X-shaped pattern (2 by 2 suture pattern). The patient's symptom improved significantly. Three months later, he fell from a bicycle. He complained of shoulder pain and limited ROM. The range of motion was as follows: forward flexion: $130^{\circ}$, abduction: $130^{\circ}$, external rotation at side: $20^{\circ}$, internal rotation at back: buttock. $X$-ray showed a protruding Versalack screw in the lateral row (Fig. 1). No evidence of medial metal anchor pull-out was found. Arthroscopy showed the protruding Versalack screw and focal partial thickness tear of the supraspinatus tendon but mostly well-healed cuff in the subacromial space. We removed the protruding anchor using the grasper and debrided the remnant
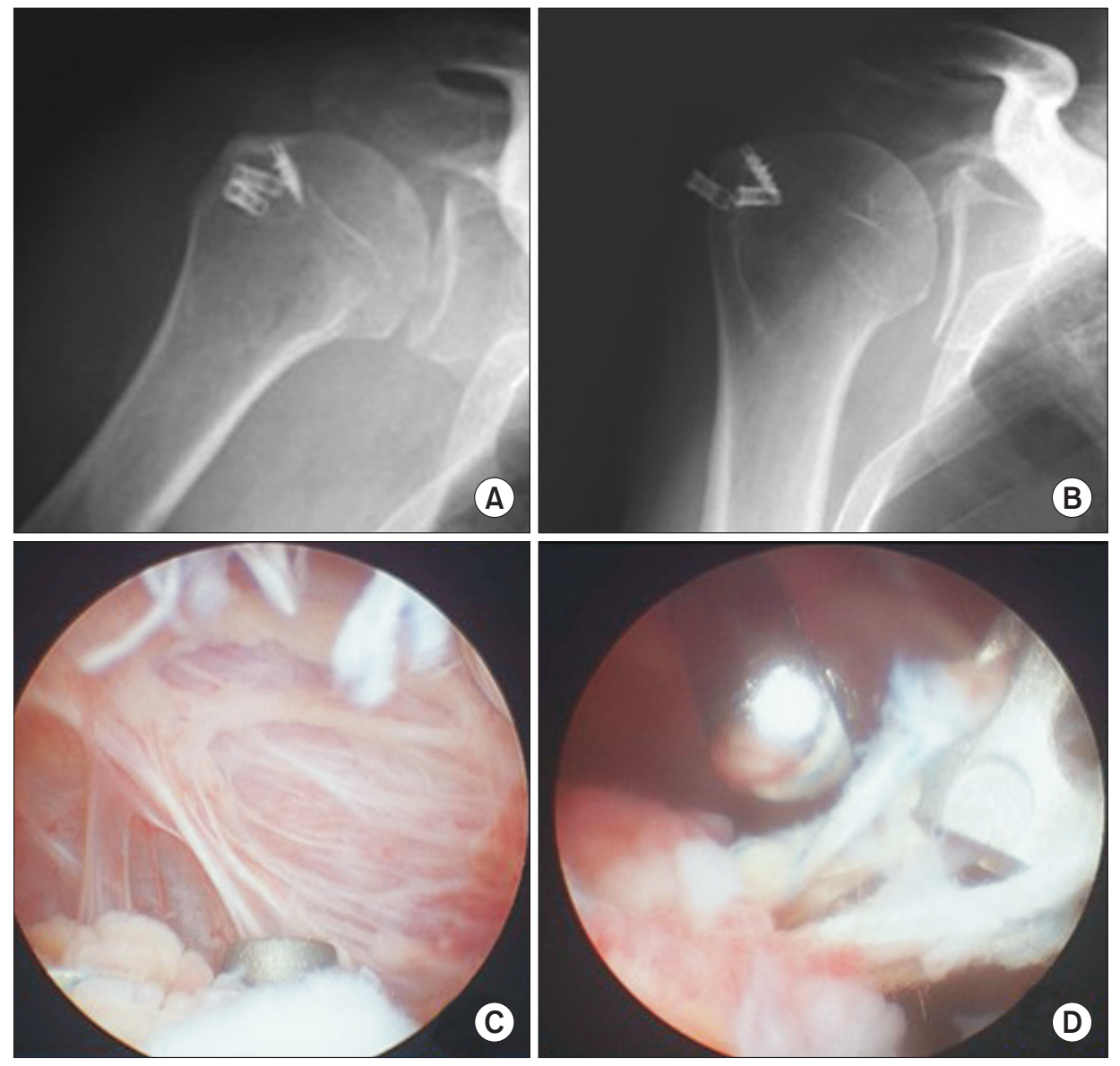

Fig. 1. (A, B) Immediate postoperative X-ray of right shoulder showed a good arrangement (medial 1 anchor, lateral 2 anchor) of the suture anchor in the suture bridge repair and postoperative 3-month X-ray revealed pull-out of the lateral row anchor from the greater tuberosity. (C, D) In the lateral decubitus position, revision arthroscopy using routine posterior portal was performed. Arthroscopic findings showed pull-out of the lateral anchor and complete healing of the rotator cuff; the protruding anchor was removed using the grasper. 
rotator cuff fraying. Two years later, he had no shoulder pain and was satisfied with the surgery (American Shoulder and Elbow Surgeons score, University of California at Los Angeles score, Simple Shoulder Test score was significantly improved) (Table 1).

\section{Case 2}

A 54-year-old female patient complained of left shoulder pain. MRI showed a partial thickness rotator cuff tear and arthroscopic findings confirmed concurrent articular- and bursalside partial thickness rotator cuff tears. Arthroscopic trans-tendon suture-bridge repair was performed for concurrent articularand bursal-side partial thickness rotator cuff tears. In the medial row, one absorbable anchor with two threads $(5.0 \mathrm{~mm}$ Twin-Fix anchor; Smith-Nephew) was inserted near the articular margin of the humeral head. After the lateral bone was prepared for anchor placement, the two limbs were passed through the eyelet of a knotless anchor (Footprint anchor; Smith-Nephew) and compressed the repaired cuff. This repair was performed in an X-shaped pattern ( 1 by 2 suture pattern). The patient was very satisfied with the rotator cuff repair.

After five months, she complained of a clicking sound in her shoulder and shoulder pain. She had no trauma history after the rotator cuff repair. The range of motion was as follows : forward flexion: $135^{\circ}$, abduction: $130^{\circ}$, external rotation at side: $20^{\circ}$, internal rotation at back: T12 level. We evaluated with a postoperative MRI, which showed suspicious partial thickness bursal sided tear and pull-out of lateral row suture anchor from the prior surgical tract in the humeral head (Fig. 2). We removed the pull-out lateral anchor but found the previously repaired cuff had healed well. The patient was satisfied after the removal of the lateral row anchor. After 2-year follow-up, she was satisfied with the results (Table 1).

Table 1. Clinical Outcomes at Last Follow-up

\begin{tabular}{|c|c|c|c|c|c|c|}
\hline \multirow{2}{*}{ Variable } & \multicolumn{2}{|c|}{ Case 1} & \multicolumn{2}{|c|}{ Case 2} & \multicolumn{2}{|c|}{ Case 3} \\
\hline & Preop & Postop & Preop & Postop & Preop & Postop \\
\hline \multicolumn{7}{|l|}{ Clinical outcome } \\
\hline ASES score & 46 & 87 & 60 & 90 & 56 & 79 \\
\hline UCLA score & 17 & 32 & 24 & 32 & 22 & 28 \\
\hline SST score & 5 & 10 & 6 & 10 & 6 & 10 \\
\hline \multicolumn{7}{|l|}{ Range of motion $\left(^{\circ}\right)$} \\
\hline FF & 130 & 150 & 135 & 155 & 130 & 150 \\
\hline Abduction & 130 & 150 & 130 & 155 & 125 & 150 \\
\hline External rotation & 20 & 30 & 20 & 40 & 15 & 30 \\
\hline Internal rotation at back & Buttock & L1 & $\mathrm{T} 12$ & $\mathrm{~T} 12$ & L3 & $\mathrm{L} 2$ \\
\hline
\end{tabular}

Preop: reoperative, Postop: postoperative, ASES: American Shoulder and Elbow Surgeons, UCLA: University of California at Los Angeles, SST: Simple Shoulder Test, FF: forward flexion.
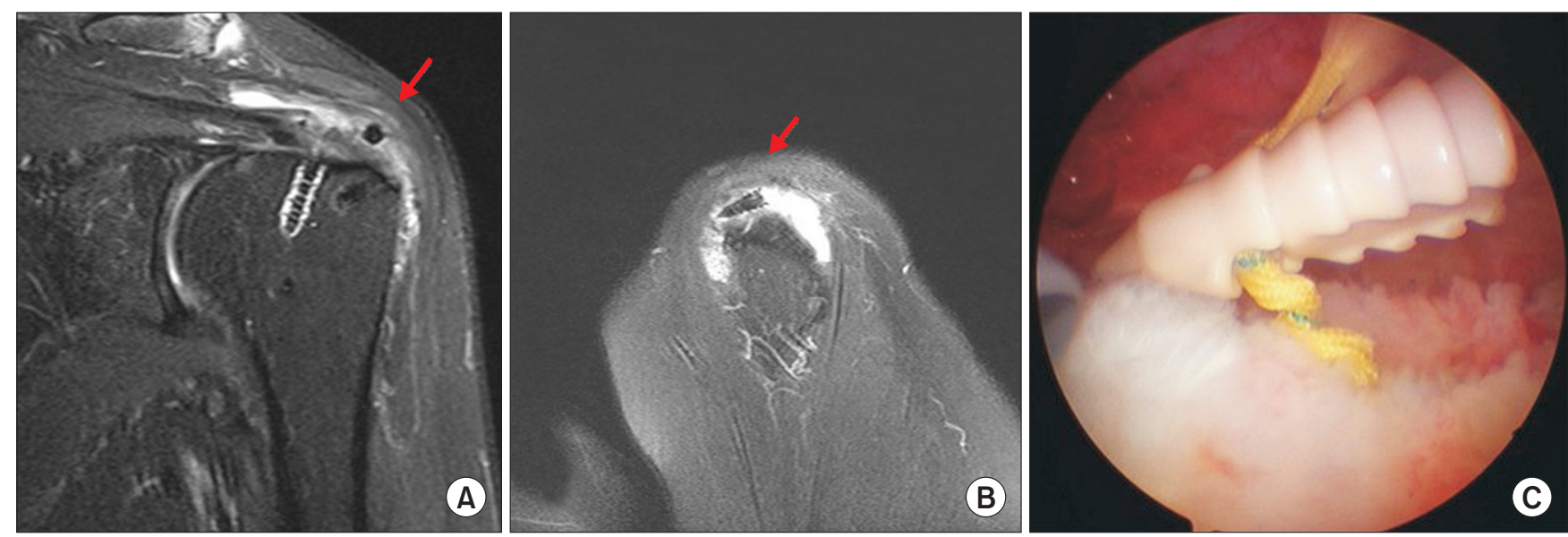

Fig. 2. Postoperative follow-up magnetic resonance imagings of left shoulder showed a suture anchor (arrows) as a dark signal intensity in the subacromial space (A, $\mathrm{B})$ and arthroscopic removal was performed (C). 


\section{Case 3}

A 59-year-old male patient had right shoulder pain. MRI showed a full-thickness tear and retraction to the humeral head (medial $1 / 3$ level) in the supraspinatus tendon. After margin convergence, a suture bridge rotator cuff repair was performed, with one suture from each medial anchor passed through the tendon and tied in a horizontal mattress pattern. One of two limbs from each of two medial anchors was pulled laterally over the tendon. After the lateral bone was prepared for anchor placement, the two limbs were passed through the eyelet of a push-lock anchor (Arthrex, Naples, FL, USA) and then the lateral anchor was placed into the prepared greater tuberosity. This repair was performed in an X-shaped pattern ( 2 by 2 suture pattern).

Six months later, severe shoulder pain and limited ROM developed without any trauma history. The range of motion was as follows: forward flexion: $130^{\circ}$, abduction: $125^{\circ}$, external rotation at side: $15^{\circ}$, internal rotation at back: L3 level. Follow-up MRI showed rotator cuff re-tear at the anterior aspect but, compared to the previous MRI, the tear was smaller. A small dark artifact was also found in the glenohumeral joint. In the arthroscopic examination, the push-lock lateral row anchor had pulled out of the original insertion site (greater tuberosity) and moved to the glenohumeral joint (Fig. 3). We removed the push-Lock anchor in the glenohumeral joint and performed revision rotator cuff repair.

On average, 3.3 suture anchors (medial 1.3, lateral 2 anchors) per shoulder were used. Three types of knotless suture anchor (push-in design) were used in our series: versalock, footprint anchor (Smith-Nephew) and push-lock anchor (Arthrex). In two and one cases, respectively, metal twin fix anchor (Smith-Nephew) and absorbable anchor (Arthrex) were used in the medial row repair. In two of three patients, MRI evaluation confirmed loosening of the lateral anchor (1 intra-articular and 1 bursal side) over time. One patient showed lateral row nonabsorbable anchor (Versalock anchor) protrusion in the greater tuberosity on a simple $\mathrm{X}$-ray. On the arthroscopic finding, two protruding anchors were found in the subacromial space and another protruding anchor was found in the glenohumeral joint. Pull-out anchors of lateral row anchors included two anterior and one posterior anchor of the greater tuberosity. None of the patients developed a wound infection after revision surgery. At the final follow-up, clinical outcomes and ROM were improved significantly after rotator cuff repair $(p<0.001)$ (Table 1$)$.

\section{Discussion}

The suture bridge repair or double row repair has recently been more commonly used due to the importance of restoring the anatomical footprint to enhance cuff healing. Suture bridge repair or double row repair provides a larger contact area for cuff repair, increases the mean contact pressure over the footprint, and also makes it biomechanically stronger ${ }^{4,5)}$ with higher structural integrity. The addition of the lateral row anchor increased the strength of cuff repair in biomechanical studies. ${ }^{2)}$ Compared with a single row repair, double row repair covered more of the footprint. ${ }^{6}$ Even though there are many controversies about the clinical outcomes between single row repair and suture bridge repair, some authors reported better clinical results and cuff integrity with double row repair compared to single row repair."

Our study reveals less satisfactory pull-out strength of the lateral row anchor in the suture bridge rotator cuff repair. We used the metal anchor and conventional absorbable suture anchor in the medial row repair among two cases and one case, respectively, and these high-pitched anchors had higher pull-out strength compared to the three types of lateral anchor (push-in design). Anchor pull-out from the bone is more common with the push-in design anchor. The pitch and pull-out strength of the medial anchor were higher than the push-in design anchor.

Kulwicki et al. ${ }^{2}$ reported that forces are transmitted through the entire portion of the tendon at its humeral fixation, loading the lateral row as well as the medial row anchors in the suture bridge repair and not interrupted at the medial anchors. They
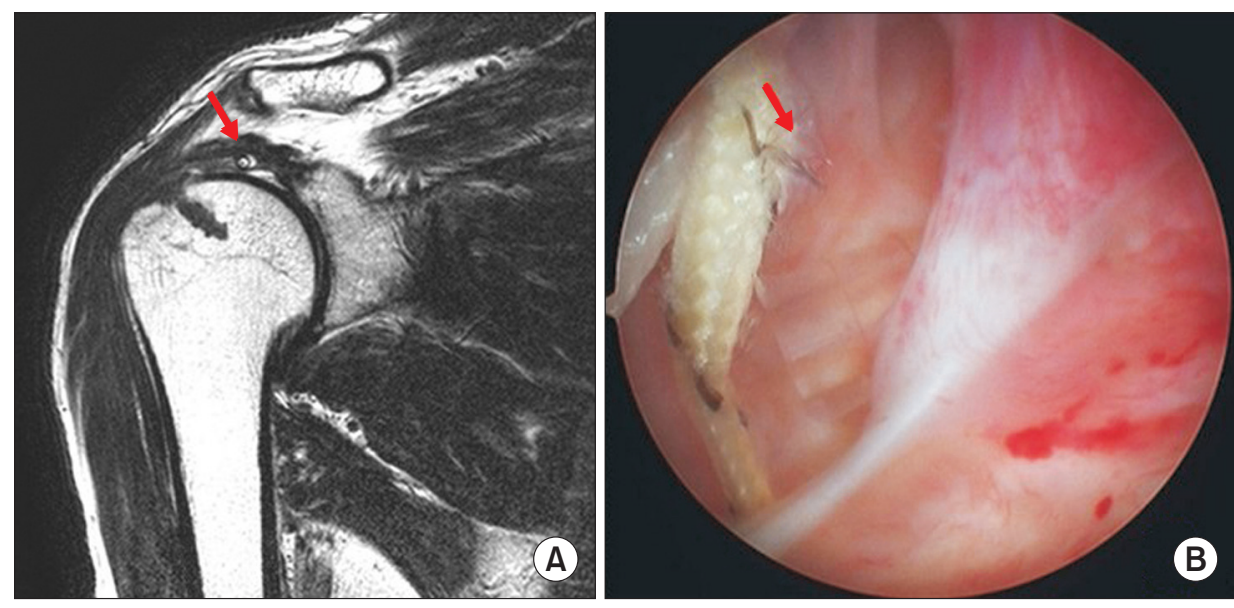

Fig. 3. Postoperative follow-up magnetic resonance imaging of right shoulder showed a suspected suture anchor (arrows) as a dark signal intensity in the glenohumeral joint (A), and arthroscopic examination confirmed that the anchor was in the glenohumeral joint (B). 
reported that there was no apparent difference in the loads borne by the medial and lateral rows for suture bridge constructs $(p=0.909)$ and that the loads borne by the medial and lateral rows in both the double-row and suture bridge repairs are similar.

One cause of lateral row anchor failure could be that the pull-out strength of the high-pitched anchor in the medial row might be higher than that of the lateral row anchor (push-in design anchor) so that the pull-out force might be transmitted to the lateral row anchor. This phenomenon is unlikely to occur due to the increased pull-out strength by the high pitch of the recent suture anchor. However, the incidence of medial or lateral anchor pull-out is believed to be higher with use of more absorbable anchors. In the second case, an absorbable medial anchor was used. This case may also be related to the number of used anchors used: a single medial anchor and two lateral anchors. A previous report ${ }^{8)}$ suggested that, after a mattress repair to the torn tendon, which placed four strands of sutures from the single medial anchor to be carried by the lateral row anchor, all traction forces on the medial anchor were then transmitted across to the lateral anchor. They proposed that this tension might be beyond the level of tension that the bone of the lateral cortex can withstand.

Arm positioning could also be one of the causes of lateral row anchor pull-out. $45^{\circ}$ internal and external rotation significantly $(p=0.032)$ increased loads on the anterior and posterior anchors by at least $125 \% .^{2}{ }^{2}$ In our cases, lateral row anchor pull-out of two anterior and one posterior anchor developed. Finally, the angle of the anchor insertion should be considered. Dead man angle $\left(45^{\circ}\right)$ was considered the most acceptable angle of the anchor insertion. However, according to the Burkhart study, the $45^{\circ}$ classical anchor insertion is weaker than a $90^{\circ}$ anchor insertion. ${ }^{9)}$ More acute angles of anchor insertion could purchase a greater quantity of better bone and provide higher fixation strength. Also when comparing standard and intracortical suture anchors, anchor motion in the bone accounted for one-third of the total displacement of the suture or anchor construct for both types of anchors and this led to increased construct displacement, which clinically might lead to repair failure. ${ }^{10)}$ In the late failure of the lateral row anchor of our series, we suggest that the medial anchor insertion might be inserted at more vertical angle $\left(90^{\circ}\right)$ rather than classical $45^{\circ}$ dead man angle. Also, patients who unintentionally experience early mobilization might develop pull-out of the lateral row anchor after rotator cuff repair. Sometimes, osteoporotic bone can also be responsible for pullout of the lateral row anchor.

In a previous study, a shoulder specimen model used during testing demonstrated loading of the cuff at $45^{\circ}$ at the medial and lateral row anchor insertion. ${ }^{2}$ If the same traction forces were transmitted across to the lateral and medial anchor such that pull-out of the lateral anchor could develop, we could change the direction of medial anchor insertion $\left(90^{\circ}\right.$ anchor insertion) in the rotator cuff tear. Acute angle anchor insertion provides less toggle while also providing higher pull-out strength rather than same direction of medial and lateral row anchor. A vertical angle anchor might also distribute the force across the healed tendon after cuff repair compared to the dead man angle anchor insertion. It is presumed that changing the direction of medial anchor insertion would reduce the incidence of lateral anchor pull-out.

In our series, pull-out of the lateral row anchor developed regardless of the anchor designs and osteoporosis. In these patients, three different anchors were used and there was no history of any osteoporosis medicine. Also, among these three patients, two patients were young and one elderly patient was an active man still using his bicycle. We suspect osteoporosis might not be directly related to the pull-out of the lateral row anchor in our series because anchor pull-out developed an average of 4.7 months (3-6 month) after cuff repair; in that time, the cuff could be completely healed.

Intraarticular foreign material can damage articular cartilage in the knee joint or shoulder joint. Severe articular damage can be caused directly by a loose intra-articular device in the knee joint. In our series, one of the lateral row anchors had shifted to the glenohumeral joint and this loose foreign body could have subsequently caused severe articular damage. If patients complain of shoulder pain several months after cuff repair, we recommend MRI evaluation for cuff re-tear and anchor failure, especially of absorbable anchors.

The limitation of this study was the small number of lateral row anchor pull-out occurrences and the retrospective analysis of lateral row anchor pull-out. Also, there was no comparison between cases with no pull-out of the lateral row anchor in intact cuff repair and delayed pull-out of the lateral row anchor. We can explain possible causes of delayed pull-out of the lateral row anchor after suture bridge repair but we cannot describe the exact cause of these delayed anchor pull-outs. Further biomechanical testing is needed to confirm the exact cause of delayed pull-out of the lateral row anchor.

In conclusion, even after complete healing of the rotator cuff tears, there are some risks of delayed pull-out of the lateral row anchor in the suture bridge rotator cuff repair. The exact causes of delayed lateral row anchor failure should be evaluated in future biomechanical studies.

\section{References}

1. Nho SJ, Slabaugh MA, Seroyer ST, et al. Does the literature support double-row suture anchor fixation for arthroscopic rotator cuff repair? A systematic review comparing doublerow and single-row suture anchor configuration. Arthroscopy. 2009;25(11):1319-28

2. Kulwicki KJ, Kwon YW, Kummer FJ. Suture anchor loading 
after rotator cuff repair: effects of an additional lateral row. J Shoulder Elbow Surg. 2010;19(1):81-5.

3. Tingart MJ, Apreleva M, Lehtinen J, Zurakowski D, Warner JJ. Anchor design and bone mineral density affect the pull-out strength of suture anchors in rotator cuff repair: which anchors are best to use in patients with low bone quality? Am J Sports Med. 2004;32(6):1466-73.

4. Milano G, Grasso A, Zarelli D, Deriu L, Cillo M, Fabbriciani C. Comparison between single-row and double-row rotator cuff repair: a biomechanical study. Knee Surg Sports Traumatol Arthrosc. 2008;16(1):75-80.

5. Meier SW, Meier JD. The effect of double-row fixation on initial repair strength in rotator cuff repair: a biomechanical study. Arthroscopy. 2006;22(11):1168-73.

6. Park MC, ElAttrache NS, Tibone JE, Ahmad CS, Jun BJ, Lee TQ. Part I: footprint contact characteristics for a transosseous- equivalent rotator cuff repair technique compared with a double-row repair technique. J Shoulder Elbow Surg. 2007;16(4): 461-8.

7. Sugaya H, Maeda K, Matsuki K, Moriishi J. Repair integrity and functional outcome after arthroscopic double-row rotator cuff repair. a prospective outcome study. J Bone Joint Surg Am. 2007;89(5):953-60.

8. Tsiouri C, Mok DH. Early pullout of lateral row knotless anchor in rotator cuff repair. Int J Shoulder Surg. 2009;3(3):63-5.

9. Burkhart SS. Suture anchor insertion angle and the deadman theory. Arthroscopy. 2009;25(12):1365.

10. Mahar A, Allred DW, Wedemeyer M, Abbi G, Pedowitz R. A biomechanical and radiographic analysis of standard and intracortical suture anchors for arthroscopic rotator cuff repair. Arthroscopy. 2006;22(2):130-5. 